\title{
Preferentially examined sentinel nodes for sentinel node navigation surgery in gastric cancer
}

\author{
YOSHIHISA YAGUCHI ${ }^{1,2}$, HIRONORI TSUJIMOTO ${ }^{1}$, SHUICHI HIRAKI ${ }^{1}$, \\ TAKASHI ICHIKURA ${ }^{1}$, JUNJI YAMAMOTO ${ }^{1}$ and KAZUO HASE ${ }^{1}$ \\ ${ }^{1}$ Department of Surgery, National Defense Medical College, Tokorozawa, Saitama 359-0042, Japan
}

Received December 8, 2014; Accepted April 15, 2015

DOI: $10.3892 / \mathrm{mco} .2015 .551$

\begin{abstract}
The intraoperative examination of the sentinel nodes (SNs) is crucial for correctly performing SN navigation surgery (SNNS). Frozen-section diagnosis is ordinarily used; however, when several SNs are being assessed in gastric cancer, which has numerous regional lymph nodes, it is difficult to examine them all correctly within the short duration of surgery. In the present study, we aimed to determine the SNs that should be preferentially examined during SNNS in gastric cancer. A total of 824 SNs were examined in 113 patients with clinically determined T1-2 gastric cancer and no apparent lymph node metastasis. We focused on the accumulation of tracers expressed by hot nodes (HNs) using the radioisotope (RI) method and green nodes (GNs) using the dye-guided method and measured the radioactivity count of the HNs (RI count). We compared these parameters between 35 metastatic and 789 non-metastatic SNs. The percentage of metastasis-positive SNs that were radioactively 'hot' and dyed green was higher compared with that of the negative SNs (89 vs. $43 \%$, respectively; $\mathrm{P}<0.01)$. The RI counts of the metastasis-positive SNs were higher compared with those of the negative SNs [median (range): $361(0-10,670)$ vs. $53(0-9,931)$, respectively; $\mathrm{P}<0.01]$. The area under the receiver operating characteristic curve of the RI count was 0.69 (95\% CI: 0.60-0.78). Therefore, when assessing several SNs, those with higher RI counts (HNs and GNs) should be preferentially examined. Further accumulation of cases is required to establish the cut-off value for the diagnosis of metastasis based on the RI count.
\end{abstract}

Correspondence to: Dr Yoshihisa Yaguchi, Present address: ${ }^{2}$ Department of Surgery, Teikyo University School of Medicine, 2-11-1 Kaga, Itabashi-ku, Tokyo 173-8605, Japan

E-mail: yaguchi@med.teikyo-u.ac.jp

Abbreviations: SN, sentinel node; SNNS, SN navigation surgery; HNs, hot nodes; GNs, green nodes; RI, radioisotope; RI count, radioactivity count of the $\mathrm{HNs}$; $\mathrm{SSs}, \mathrm{SN}$ stations; H\&E, hematoxylin and eosin; OSNA, one-step nucleic acid amplification

Key words: gastric cancer, sentinel node, sentinel node navigation surgery, intraoperative examination

\section{Introduction}

After Morton et al reported the usefulness of intraoperative lymphatic mapping for melanoma in the early 1990s (1), the sentinel node (SN) concept has been widely accepted for the treatment of various types of cancer, including breast, gastric and head and neck cancer (1-5). Sentinel node navigation surgery (SNNS) is currently a standard procedure for early-stage melanoma and breast cancer. SNNS for gastric cancer, however, is currrently in the research phase, having been investigated at only a few institutes to date $(6,7)$. Furthermore, two large prospective multicenter trials in Japan have reported conflicting results regarding the clinical application of SN biopsies for gastric cancer $(8,9)$, suggesting that it is difficult to precisely detect $\mathrm{SNs}$ in gastric cancer compared with melanoma or breast cancer.

A hindrance in establishing SNNS as a standard procedure in gastric cancer is the complexity of the lymphatic flow and the numerous regional lymph nodes in the stomach. The number of SNs in gastric cancer is higher compared with that in melanoma or breast cancer; for example, the mean number of SNs in melanoma or breast cancer is 1-3 (10), whereas the mean number in gastric cancer is 4-7 (7). Moreover, when several SNs are detected, it may be difficult to determine which SN should be examined during surgery.

In the present study, we aimed to determine which SNs should be preferentially examined during gastric cancer surgery in order to detect metastatic SNs.

\section{Patients and methods}

Patients. In total, 824 SNs from 113 patients with clinically determined T1-2 gastric cancer with no apparent lymph node metastases were included in this study. We attempted to detect SNs in these patients through the use of radioisotope (RI) and dye methods during the period between November, 2002 and August, 2011. There were a total of 35 metastasis-positive and 789 metastasis-negative SNs.

SN identification. SNs were identified by a combination of the RI and dye methods and classified as hot nodes (HNs) and/or green nodes (GNs) as follows: In the RI method, $0.5 \mathrm{ml}$ of ${ }^{99 m}$ Tc-tin colloid solution was injected into each of four sites surrounding the tumor on the day prior to surgery and HNs 
Table I. Demographic data.

\begin{tabular}{lc}
\hline Characteristics & Patient no. $(\mathrm{n}=113)$ \\
\hline Age, years & \\
Mean \pm SD & $64 \pm 11$ \\
Gender & \\
Male & 77 \\
Female & 36 \\
Histology & \\
Differentiated & 68 \\
Undifferentiated & 45 \\
Depth & \\
Mucosa & 52 \\
Submucosa & 43 \\
Muscularis propria & 14 \\
Subserosa & 3 \\
Serosa & 1 \\
LN metastasis ${ }^{\mathrm{a}}$ & \\
N0 & \\
N1 & \\
N2 & \\
Tumor size, cm & \\
Mean \pm SD & \\
\hline Japanese Gastric Cancer Association, 14th edition. SD, standard \\
deviation; LN, lymph node. & \\
\hline
\end{tabular}

were defined as lymph nodes with a radioactivity of $\geq 10$ counts per $10 \mathrm{sec}$. In the dye method, $1 \mathrm{ml}$ of $1.25 \%$ indocyanine green solution was injected into each of four sites surrounding the tumor and GNs were defined macroscopically.

Five minutes following dye injection, we attempted to determine the SN stations (SSs) where SNs were distributed. We then dissected the remaining lymph node stations, which was required for the preoperatively planned dissection. The SNs were examined on a back table in the operating room.

Tracer accumulation and radioactivity count of the HNs (RI count). We focused on the accumulation of tracers expressed by HNs and GNs, the RI count and the size of the SNs. To establish a cut-off value for the RI count, we evaluated the diagnostic characteristics from the receiver operating characteristic (ROC) curve of the RI count. We also determined the number of SNs that must be examined to detect metastatic SNs when HNs and GNs with high RI counts are preferentially examined.

The SN biopsies and SNNS procedures reported in this study were reviewed and approved by the Institutional Review Board of the National Defense Medical College (Saitama, Japan) and written informed consent was obtained from all the patients prior to conducting the procedures for SN identification.

Statistical analysis. All the data were analyzed using Dr. SPSS II software for Windows (SPSS Japan Inc., Tokyo,
Table II. Number of SNs and surgical procedure.

\begin{tabular}{lc}
\hline Variables & Values \\
\hline Total cases, no. & 113 \\
Total SNs, no. & 824 \\
SN no., median (range) & $6(1-22)$ \\
HNs & $4(0-22)$ \\
GNs & $4(0-17)$ \\
HNs and GNs & $2(0-14)$ \\
SS no. (median, range) & $2(1-4)$ \\
Surgical procedure, no. & \\
Partial gastrectomy & 9 \\
Sleeve gastrectomy & 31 \\
Pylorus-preserving gastrectomy & 22 \\
Distal gastrectomy & 35 \\
Proximal gastrectomy & 10 \\
Total gastrectomy & 6
\end{tabular}

SNs, sentinel nodes; HNs, hot nodes; GNs, green nodes; SSs, SN stations.

Japan). Data are expressed as means \pm standard deviations and median (range). The Mann-Whitney U test and Chi-square test were used for comparisons between the metastatic and non-metastatic groups. P-values of $<0.05$ were considered to indicate statistically significant differences.

\section{Results}

Demographic data. Of the 113 patients with cT1-2 gastric cancer, $4(3.5 \%)$ were diagnosed as $\geq \mathrm{pT} 3$, whereas $23(20 \%)$ exhibited lymph node metastases (Table I).

Number of SNs and surgical procedures. A total of 824 lymph nodes were examined. The median number (range) of SNs, HNs and GNs was 6 (1-22), 4 (0-22) and 4 (0-17), respectively (Table II). The surgical procedures included SNNS with a negative SN biopsy (partial gastrectomy, 9 cases; and sleeve gastrectomy, 31 cases; Table II).

Accumulation of tracers and SN size. We compared the accumulation of tracers and size of lymph nodes between the metastasis-positive and metastasis-negative SNs. The ratio of HNs in metastasis-positive SNs was significantly higher compared with that in negative SNs (91 vs. 67\%, respectively; $\mathrm{P}<0.01)$. The ratio of GNs was also significantly higher in metastasis-positive compared with that in negative SNs (97 vs. $76 \%$, respectively; $\mathrm{P}<0.01)$. The most significant difference between the two groups was observed in the ratio of the combination of HNs and GNs ( 89 vs. 43\%, respectively; $\mathrm{P}<0.01$ ). The RI count of the metastatic SNs was significantly higher compared with that of the negative SNs [median (range): $361(0-10,670)$ vs. 53 (0-9,931), respectively; $\mathrm{P}<0.01]$. There was no significant difference in SN size between the two groups [median (range): 4.0 (1.7-15.0) vs. 4.0 (0.5-20.0) mm, respectively; Table III). 
Table III. Accumulation of tracers and size of SNs.

\begin{tabular}{lccc}
\hline SN metastasis & Positive $(\mathrm{n}=35)$ & Negative $(\mathrm{n}=789)$ & P-value \\
\hline Hot nodes, no. $(\%)$ & $32(91)$ & $528(67)$ & $<0.01$ \\
Green nodes & $34(97)$ & $601(76)$ & $<0.01$ \\
Hot and green nodes & $31(89)$ & $53(0-9,931)$ & $<0.01$ \\
RI count ${ }^{\mathrm{a}}$, median (range) & $361(0-10,670)$ & $4.0(0.5-20.0)$ & $<0.01$ \\
SN size $(\mathrm{mm})$, median (range) & $4.0(1.7-15.0)$ & 0.40 \\
\hline
\end{tabular}

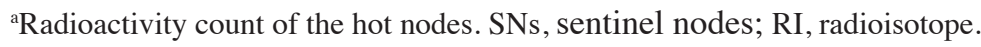

Table IV. Diagnostic characteristics of the radioactivity count of the hot nodes (RI count).

\begin{tabular}{lcccr}
\hline RI count & $\begin{array}{c}\text { Sensitivity } \\
(95 \% \mathrm{CI})\end{array}$ & $\begin{array}{c}\text { Specificity } \\
(95 \% \mathrm{CI})\end{array}$ & $\begin{array}{c}\text { Positive likelihood } \\
\text { ratio }(95 \% \mathrm{CI})\end{array}$ & $\begin{array}{c}\text { Negative likelihood } \\
\text { ratio }(95 \% \mathrm{CI})\end{array}$ \\
\hline 100 & $0.71(0.53-0.85)$ & $0.58(0.54-0.61)$ & $1.68(1.31-2.16)$ & $0.50(0.28-0.90)$ \\
200 & $0.64(0.46-0.79)$ & $0.68(0.64-0.71)$ & $1.99(1.48-2.68)$ & $0.53(0.32-0.87)$ \\
300 & $0.61(0.42-0.76)$ & $0.75(0.72-0.78)$ & $2.46(1.78-3.40)$ & $0.52(0.33-0.83)$ \\
\hline
\end{tabular}

$\mathrm{RI}$, radioisotope; CI, confidence interval.

ROC curve and diagnostic characteristics of the RI count. The area under the ROC curve of the RI count was 0.69 [95\% confidence interval (CI): 0.60-0.78; Fig. 1]. We set the cut-off values at 100, 200, and 300 in view of the inflection point of the ROC curve. The sensitivity was $71 \%$ when the cut-off value was set at 100 and $61 \%$ when the cut-off value was set at 300 (Fig. 1, Table IV).

Examinations required to detect metastatic SNs. There were 19 metastatic SNs cases in this study. Although there were three cases with insufficient data (nos. 7, 9 and 15), it was clear that, in order to detect metastatic SNs, we only had to preferentially examine 1-2 HNs and GNs with high RI counts, with the exception of case no. 8 (Table V).

\section{Discussion}

In this study, we attempted to determine the priority with which SNs should be examined during SNNS for gastric cancer. During surgery, it is difficult to examine and assess multiple SNs promptly and accurately. Therefore, it is crucial to determine which SNs are the best candidates for metastasis based on size and the accumulation of tracers to ensure successful SNNS in gastric cancer.

We also investigated the possibility of establishing a diagnosis of metastasis based on the RI count. Although metastatic SNs exhibited higher RI counts compared with non-metastatic SNs, the area under the ROC curve and the diagnostic characteristics of the RI count were insufficient for setting firm cut-off values; thus, further evaluation of additional cases is required to explore this methodology (Fig. 1, Table IV). The diagnostic role of radioactivity in SNs for breast cancer is, to a certain degree, established (11-13); however, although the SNs

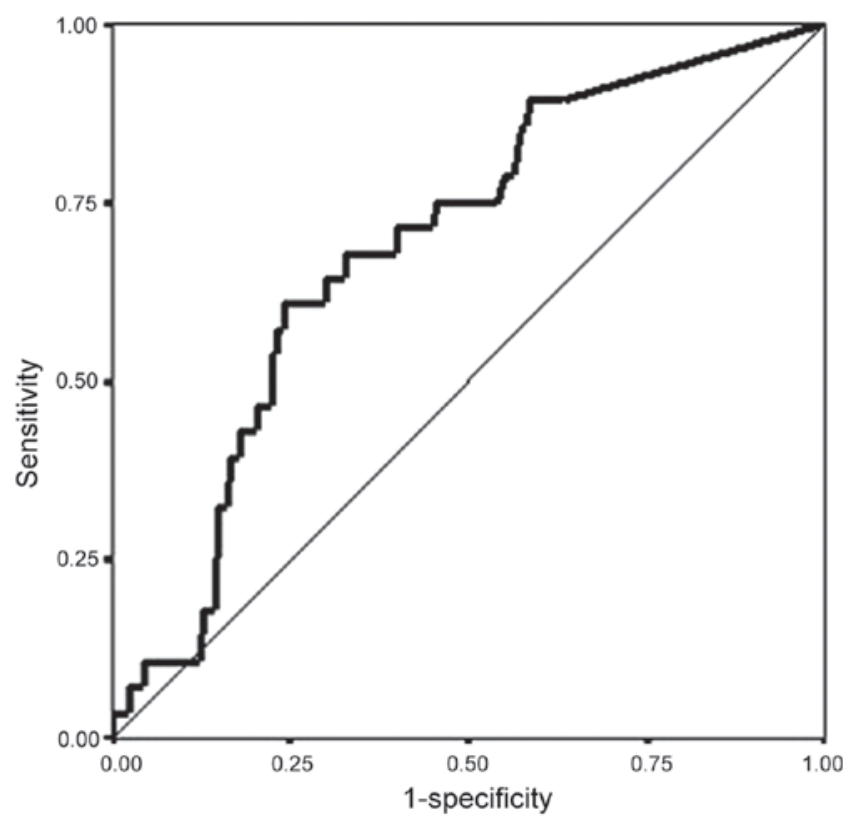

Figure 1. Receiver operating characteristic (ROC) curve of RI counts The area under the ROC curve of the RI count was 0.69 (95\% confidence interval: 0.60-0.78).

with the highest counts are positive in the majority of breast cancer patients with multiple SNs, a consistent and relatively high RI count does not predict SN positivity in all breast cancer patients (11-13). Similar results have been reported in melanoma and head and neck cancer $(14,15)$.

Although these findings indicate that it is difficult to diagnose metastasis by RI count, RI counts may be of value in the preferential selection of SNs. Based on the review 
Table V. Examinations required for the detection of metastatic SNs.

Case

number
Total SNs
Number of examinations required to detect metastatic $\mathrm{SNs}^{\mathrm{a}}$

\begin{tabular}{rrrr}
\hline 1 & 2 & 1 & 1 \\
2 & 3 & 1 & 2 \\
3 & 10 & 2 & 1 \\
4 & 7 & 1 & 1 \\
5 & 5 & 2 & 1 \\
6 & 16 & 5 & 2 \\
7 & 6 & 4 & Not clear \\
8 & 8 & 1 & 7 or 8 \\
9 & 21 & 1 & Not clear \\
10 & 18 & 2 & 1 \\
11 & 11 & 1 & 1 \\
12 & 9 & 3 & 2 \\
13 & 5 & 1 & 1 \\
14 & 13 & 1 & 1 \\
15 & 16 & 1 & Not clear \\
16 & 3 & 3 & 1 \\
17 & 6 & 3 & 1 \\
18 & 1 & 1 & 1 \\
19 & 7 & 1 & 1 \\
\hline
\end{tabular}

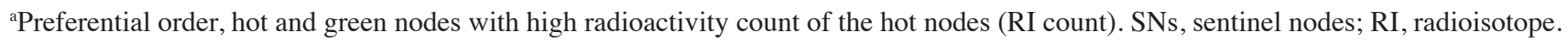

of metastatic SN cases in the present study, we concluded that, in order to determine the presence or absence of metastatic SNs, only 1-2 SNs must be examined during surgery, even if $>10$ SNs are detected; however, it should be noted that 3 cases presented with insufficient data, whereas 1 case (no. 8) required the examination of 7-8 nodes, thus contradicting this theory (Table V). Case no. 8 had advanced gastric cancer with T2 of the posterior side on the upper portion with a $30-\mathrm{mm}$ tumor and had metastasis with GNs in all 8 SNs (6 hot and green nodes and 2 GNs) distributed around the right paracardial and lesser curvature. It has not been elucidated why this case only metastasized to the GNs; however, this suggests that it is crucial to limit SNNS to early gastric cancer.

There has been some debate over the actual procedures for the clinical application of SNNS in gastric cancer, including the type of tracer to be used, the injection site, how to detect and harvest SNs and how to detect metastatic SNs (16). As regards the detection of metastatic SNs, researchers tend to focus on diagnostic methods, such as molecular techniques, rather than frozen section diagnoses with hematoxylin and eosin (H\&E), which tend to be inaccurate. We previously reported that the one-step nucleic acid amplification (OSNA) method, which is a semi-automated molecular-based rapid diagnostic method, has the same diagnostic ability as the final H\&E-based histopathological examination and that it should be applied for intraoperative diagnoses in SNNS for gastric cancer (17). The OSNA method may be used to diagnose $\mathrm{SN}$ metastasis within $30 \mathrm{~min}$, although it is difficult to simultaneously examine numerous SNs during surgery; a maximum of 4 SNs may be examined using the
OSNA measurement equipment that is currently available. Therefore, when assessing several SNs, we recommend that SNs that have high RI counts with both 'hot' and 'green' status are preferentially examined.

Although multicenter trials with a larger number of cases are required to confirm our results, we are convinced that the prioritization described herein will speed up intraoperative diagnosis, enabling the wider application of SNNS in clinical practice. However, further accumulation of cases is required to set the cut-off values for the diagnosis of metastasis based on the RI count.

\section{References}

1. Morton DL, Wen DR, Wong JH, Economou JS, Cagle LA Storm FK, Foshag LJ and Cochran AJ: Technical details of intraoperative lymphatic mapping for early stage melanoma. Arch Surg 127: 392-399, 1992.

2. Krag D, Weaver D, Ashikaga T, et al: The sentinel node in breast cancer - a multicenter validation study. N Engl J Med 339: 941-946, 1998.

3. Veronesi U,Paganelli G, Viale G, et al: A randomized comparison of sentinel-node biopsy with routine axillary dissection in breast cancer. N Engl J Med 349: 546-553, 2003.

4. Ross G, Shoaib T, Soutar DS, Camilleri IG, Gray HW, Bessent RG, Robertson AG and MacDonald DG: The use of sentinel node biopsy to upstage the clinically N0 neck in head and neck cancer. Arch Otolaryngol Head Neck Surg 128: 1287-1291, 2002.

5. Ross GL, Soutar DS, Gordon MacDonald D, et al: Sentinel node biopsy in head and neck cancer: preliminary results of a multicenter trial. Ann Surg Oncol 11: 690-696, 2004.

6. Ohdaira H, Nimura H, Mitsumori N, Takahashi N, Kashiwagi H and Yanaga K: Validity of modified gastrectomy combined with sentinel node navigation surgery for early gastric cancer. Gastric Cancer 10: 117-122, 2007. 
7. Ichikura T, Sugasawa H, Sakamoto N, Yaguchi Y, Tsujimoto H and Ono S: Limited gastrectomy with dissection of sentinel node stations for early gastric cancer with negative sentinel node biopsy. Ann Surg 249: 942-947, 2009.

8. Miyashiro I, Hiratsuka M, Sasako M, Sano T, Mizusawa J, Nakamura K, Nashimoto A, Tsuburaya A and Fukushima N; Gastric Cancer Surgical Study Group (GCSSG) in the Japan Clinical Oncology Group (JCOG): High false-negative proportion of intraoperative histological examination as a serious problem for clinical application of sentinel node biopsy for early gastric cancer: final results of the Japan Clinical Oncology Group multicenter trial JCOG0302. Gastric Cancer 17: 316-323, 2014.

9. Kitagawa Y, Takeuchi H, Takagi Y, et al: Sentinel node mapping for gastric cancer: a prospective multicenter trial in Japan. J Clin Oncol 31: 3704-3710, 2013.

10. Morton DL, Thompson JF, Essner R, et al: Validation of the accuracy of intraoperative lymphatic mapping and sentinel lymphadenectomy for early-stage melanoma: a multicenter trial. multicenter selective lymphadenectomy trial group. Ann Surg 230: 63-67, 1999.

11. Martin RC, Fey J, Yeung H, Borgen PI and Cody HS III: Highest isotope count does not predict sentinel node positivity in all breast cancer patients. Ann Surg Oncol 8: 592-597, 2001.
12. Bourgeois P, Nogaret JM, Veys I, Hertens D, Dagnelie J, Vanhaudenaerde C, Verdebout JM and Larsimont D: How 'hot' is the pathologically positive sentinel lymph node in breast cancer patients? Nucl Med Commun 24: 513-518, 2003.

13. Morota S, Koizumi M, Koyama M, et al: Radioactivity thresholds for sentinel node biopsy in breast cancer. Eur J Surg Oncol 32: 1101-1104, 2006.

14. Carlson GW, Murray DR, Thourani V, Hestley A and Cohen C: The definition of the sentinel lymph node in melanoma based on radioactive counts. Ann Surg Oncol 9: 929-933, 2002.

15. Kovacs AF, Dobert N, Walendzik H, Zaplatnikov K and Landes CA: The diagnostic role of radioactivity in sentinel nodes in oral and oropharyngeal cancer. Cancer Biother Radiopharm 21: 535-543, 2006

16. Miyashiro I: What is the problem in clinical application of sentinel node concept to gastric cancer surgery? J Gastric Cancer 12: 7-12, 2012.

17. Yaguchi Y, Sugasawa H, Tsujimoto H, et al: One-step nucleic acid amplification (OSNA) for the application of sentinel node concept in gastric cancer. Ann Surg Oncol 18: 2289-2296, 2011. 